[Chem. Pharm. Bull.

35(10)4346-4350(1987)

\title{
Preparation and Evaluation of Sustained Release Tablets Prepared with $\alpha$-Starch ${ }^{11}$
}

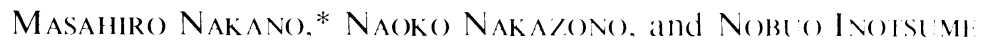

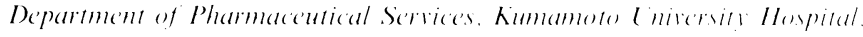

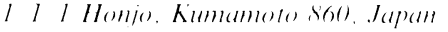

(Received March 16. 1987)

\begin{abstract}
A compressed tablet prepared from $x$-starch adsorbed water from the releane medium and formed a gel. starting from the tablet surface. Theophylline was released slowly through the gelled layer. Release rates of theophylline from tablets prepared with $x$-starches from different soures were different. Release rates of theophylline from the tablets were faster in an acidie medium than in a neutral medium. The release rate of theophylline was faster in at medium containing $x$-amblase than in a medium without the enzyme.

Salivary levels were lower but were sustained for longer following oral atdministration of an \%starch tablet than those after administration of a rapidly dissolving tablet.
\end{abstract}

Keywords $\quad x$-starch: gel: sustained release: $y$-amylase: theophylline: saliant kel

\section{Introduction}

Examination of natural gelforming materials as matrices for the preparation of sustained release tablets is important from the viewpoint of employing economical and safe materials for designing pharmaceutical products. We have already reported on the possible use of hydroxypropylcellulose, ${ }^{2}$ seaweed extracts and seed gums ${ }^{31}$ to prepare sustained release theophylline tablets. Daly et al. $^{+1}$ reported on the release characteristics of a model drug from Synchron ${ }^{\circledR}$, a specially treated form of hydroxypropylmethylcellulose.

Although natural starch is not soluble in water, it becomes water-soluble when it is heated in water and then quickly spray-dried or drum-dried. This pregelatinized starch is called $\alpha$-starch. In the present study, we examined the gel-forming properties of $y$-starches to assess the suitability of the starches for the preparation of sustained-release tablets.

\section{Experimental}

Materials $\quad \alpha$-Starches were generously supplied by Matsuya Kagaku. Itami. Hyogo Prefecture. While potato starch and low-substituted hydroxypropyleellulose were supplied by Hoci Yakko. () sakit and Shin-etsu (hemicals. Tokyo, respectively. Theophylline (anhydrous), 8-chlorotheophylline, and y-amylase were purchased from Wako Pure Chemical Ind., Osaka, while Neo-Amylase Test was purchased from Daiichi (hemicals. Tokyo. All other chemicals used for the preparation of release media and the mobile phase and for extraction were of analytical grade.

Preparation of Tablets Tablets, $13 \mathrm{~mm}$ in diameter, were prepared by compressing directly $500(0) \quad 40(0) \mathrm{mg}$ of a $1: 1$ mixture of theophylline and each $\alpha$-starch at $200 \mathrm{~kg} \mathrm{~cm}^{2}$ ats reported carlier. ${ }^{31}$ Rapidly dissolving tablets were prepared by compressing directly a $1: 1$ mixture of theophylline and low-substituted hydroxypropylcellulose. Completion of dissolution of theophylline from rapidly dissolving tablet in about 10 min has already been shown "

Release Studies - Procedures reported earlier ${ }^{31}$ were employed except that JP X disintegration test medium No. 2 containing $\alpha$-amylase at $5 \mu \mathrm{g} / \mathrm{ml}$ was used in some release studies. $x$-Amylase activity was determined by employing the Neo-Amylase Test, based on the blue-starch method. Average $x$-amylase activity was 1694 II 1 . which is close to the median $x$-amylase activity in pancreatic juice.

Measurement of Salivary Levels in Human Volunteers Four healthy volunters. one male and three fimales. of 23.47 years of age participated in the in vivo study. To eliminate ingestion of theophylline from other soures. the subjects were told to abstain from any drinks containing xanthines. 
After overnight fasting, a single 200 -mg dose of a rapidly dissolving tablet or a potato $\alpha$-starch tablet was administered with $200 \mathrm{ml}$ of water. The mouth was quickly rinsed with water to remove any theophylline adhering to the oral mucosa.

Saliva samples were collected at appropriate intervals up to $50 \mathrm{~h}$. A small amount of citric acid, a salivary flow stimulant, was put on the tongue and held in the mouth for $1 \mathrm{~min}$, then about 2-ml of saliva was collected in a test tube and kept frozen until analysis. No food was taken for $4 \mathrm{~h}$ post-dose but food and drink were taken $a d$ lib. after $4 \mathrm{~h}$. A crossover design was used and a minimum interval of 1 week was allowed between trials.

Analysis of Theophylline Amounts of theophylline released into release media were determined with a spectrophotometer. Theophylline in saliva was analyzed by using reverse-phase high-performance liquid chromatography ${ }^{6)}$ employing 8-chlorotheophylline as an internal standard.

Pharmacokinetic Analysis-Moment analysis ${ }^{7)}$ was employed to calculate the area under the salivary concentration curve $\left(A U C_{0-\infty}\right)$ and the mean residence time $(M R T)$.

\section{Results and Discussion}

\section{Behavior in Aqueous Media}

Natural starch such as potato starch was not soluble in water and a compressed tablet prepared from natural starch disintegrated when it was placed in water. When a compressed tablet prepared from $\alpha$-starch such as potato $\alpha$-starch was placed in water, it did not disintegrate but it adsorbed water to form a gel starting from the tablet surface.

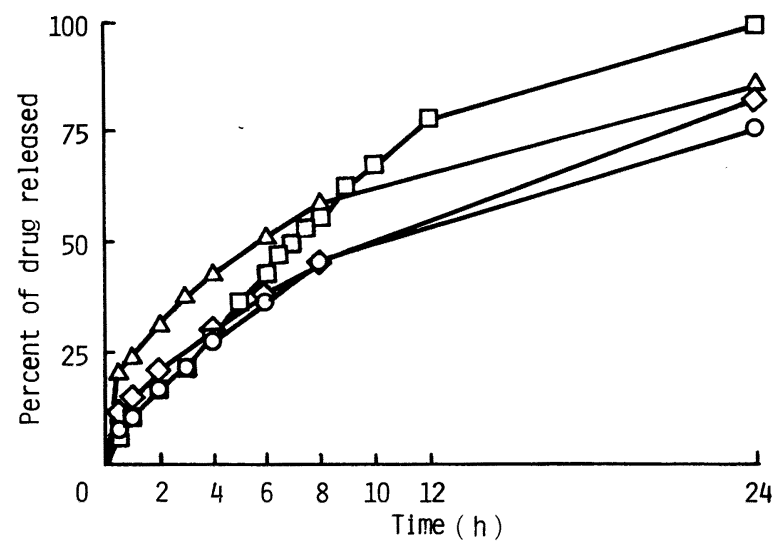

Fig. 1. Release Profiles of Theophylline from $\alpha$-Starch Tablets $\diamond$, corn; $\bigcirc$, potato; $\square$, waxy corn; $\triangle$, wheat. Medium No. $1, n=3$.
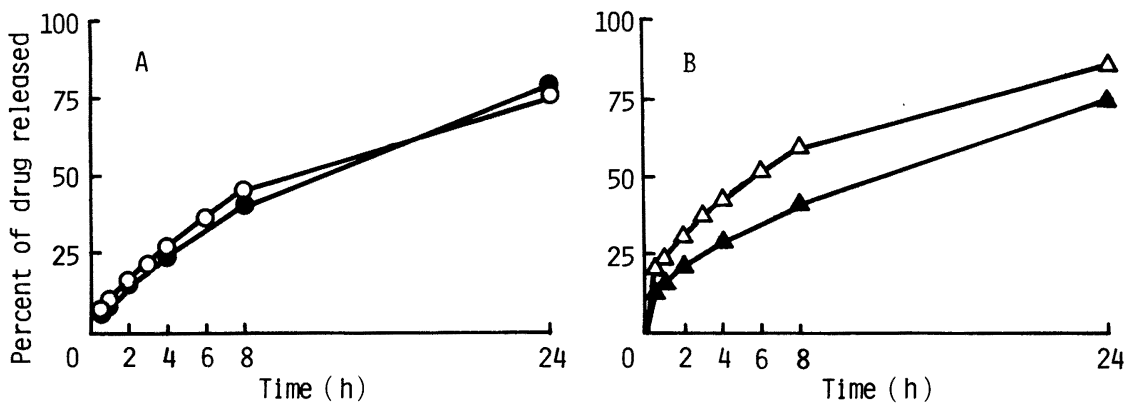

Fig. 2. Effect of $\mathrm{pH}$ Value of Release Media on Release of Theophylline from $\alpha$ Starch Tablet

A (Potato $\alpha$-starch): $\bigcirc$, medium No. 1; medium No. 1; $\boldsymbol{\Delta}$, medium No. 2 . $n=3$. 


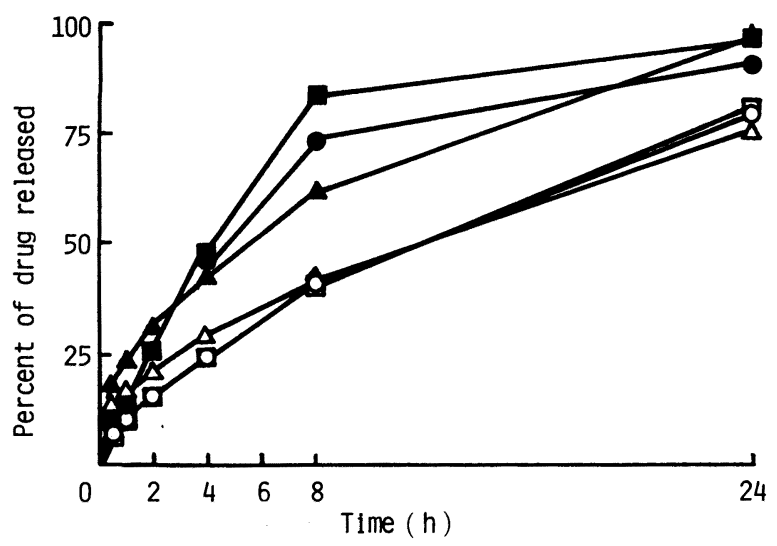

Fig. 3. Effect of $\alpha$-Amylase on Release of Theophylline from Three $\alpha$-Starch Tablets

$\bigcirc$, potato without $\alpha$-amylase; $\bullet$, potato with $\alpha$-amylase; $\square$, waxy corn without $\alpha$ amylase; $\boldsymbol{\square}$, waxy corn with $\alpha$-amylase; $\Delta$, wheat without $\alpha$-amylase; $\boldsymbol{\Delta}$, wheat with $\alpha$ amylase. Medium No. 2. $n=3$.

\section{Effect of Source of Starch}

Release profiles of theophylline in JP disintegration medium No. 1 from tablets prepared from four $\alpha$-starches from different sources are shown in Fig. 1. The drug was released fastest from the tablet prepared from $\alpha$-starch of wheat origin while it was released most slowly from the tablets prepared from $\alpha$-starch of potato or corn origin. These difference may be attributed to differences in hydration (i.e. gelation) rates due to differences in starch structure and contents of amylose and amylopectin.

\section{Effects of Release Media}

The effects of the media on the release profiles of theophylline from tablets prepared with $\alpha$-starches of potato and wheat origin are shown in Fig. $2 \mathrm{~A}$ and $2 \mathrm{~B}$, respectively. Rates of release tended to be faster in the acidic medium (medium No. 1, pH 1.2) than in the neutral medium (medium No. 2, $\mathrm{pH}$ 6.8). The effect of medium $\mathrm{pH}$ was more pronounced with $\alpha$ starch of wheat origin than with that of potato origin. A significant effect of medium $\mathrm{pH}$ was also observed in $\alpha$-starch of waxy corn origin (not shown). The difference may be attributed to differences in the susceptibility of the starch molecules to acid hydrolysis.

\section{Effect of $\alpha$-Amylase}

The effect of $\alpha$-amylase in the release media on the release patterns of theophylline from tablets is shown in Fig. 3. In tablets prepared from three types of $\alpha$-starches, the drug release in media containing $\alpha$-amylase was faster than that in media without the enzyme. The increase in release rates may be attributed to the destruction of starch matrices caused by hydrolysis of starch molecules with $\alpha$-amylase.

\section{In Vivo Study}

Based on the effects of gastrointestinal $\mathrm{pH}$ and a digestive enzyme on the release patterns of theophylline from compressed tablets, potato $\alpha$-starch was selected for the in vivo study. Salivary levels of theophylline after oral administration of a rapidly dissolving tablet or a sustained-release matrix tablet prepared with $\alpha$-starch of potato origin are shown in Fig. 4. Salivary levels rose quickly to reach the maximum concentration at $1.4 \mathrm{~h}$ following administration of the rapidly dissolving tablet, whereas they rose slowly to reach the maximum concentration at $5 \mathrm{~h}$ following administration of the sustained release tablet. 


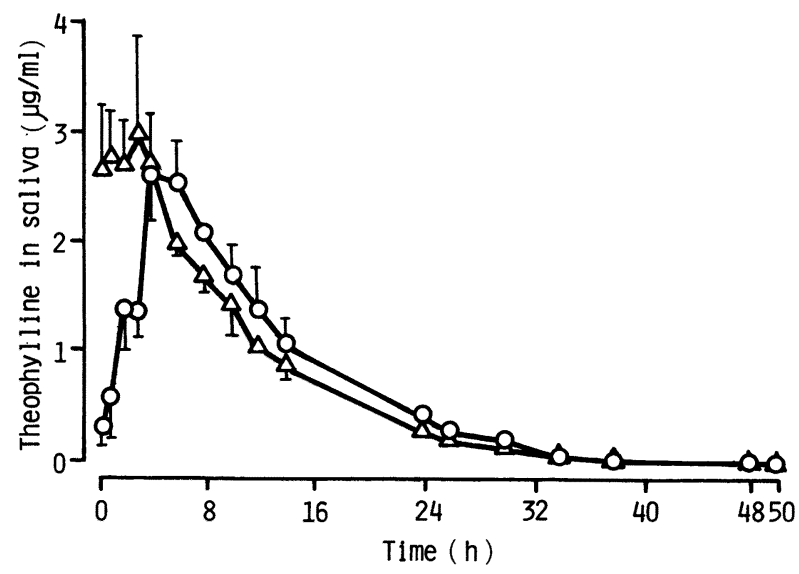

Fig. 4. Salivary Levels of Theophylline after Oral Administration of Potato $\alpha$ Starch Tablet and Rapidly Dissolving Tablet in Volunteers

$\triangle$, rapidly dissolving tablet; $O$, potato $\alpha$-starch tablet. Mean \pm S.E.M. $n=4$.

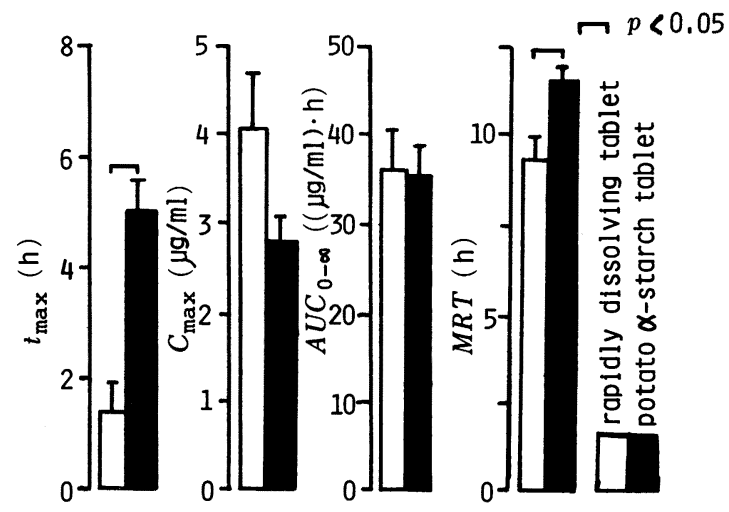

Fig. 5. Comparison of Bioavailability Parameters between Potato $\alpha$-Starch Tablet and Rapidly Dissolving Tablet

Figure 5 shows bioavailability parameters for two theophylline preparations. Although $t_{\max }$ and $M R T$ values were significantly different among the two, $A U C_{0-\infty}$ and $C_{\max }$ values were not significantly different. This observation indicates that theophylline was absorbed to a similar extent despite the difference in release rates from the tablets and resultant difference in absorption patterns.

\section{Conclusion}

The present results suggest that

1) Compressed $\alpha$-starch tablets form a gel by adsorbing water from aqueous media.

2) Some $\alpha$-starches are more susceptible to acid hydrolysis than others.

3) All $\alpha$-starches are susceptible to enzymatic hydrolysis with $\alpha$-amylase but the degree of susceptibility is variable.

4) $\alpha$-Starches can be used as a matrix material for sustained-release tablets.

Acknowledgements We are grateful to Miss Ryoko Honda and Miss Miwako Kaneshige for their technical assistance. A part of this work was supported by a Grant-in-Aid for Scientific Research (No. 58570905) from the Ministry of Education, Science, and Culture, Japan. 


\section{References and Notes}

1) Presented at the 105th Annual Meeting of the Pharmaceutical Society of Japan, April 1985 in Kanazawa.

2) M. Nakano, N. Ohmori, A. Ogata, K. Sugimoto, Y. Tobino, R. Iwaoku, and K. Juni, J. Pharm. Sci., 72, 378 (1983).

3) M. Nakano and A. Ogata, Chem. Pharm. Bull., 32, 782 (1984).

4) P. B. Daly, S. S. Davis, M. Frier, J. Hardy, J. Kennerley, and C. G. Wilson, J. Pharm. Pharmacol., 33, 47P (1981).

5) N. Nakazono, N. Inotsume, Y. Tobino, R. Iwaoku, and M. Nakano, Jpn. J. Clin. Pharmacol. Ther., 16, 401 (1985).

6) M. Nakano, Y. Nakamura, K. Juni, and T. Tomitsuka, J. Pharmacobio-Dyn., 3, 702 (1980).

7) K. Yamaoka, T. Nakagawa, and T. Uno, J. Pharmacokinet. Biopharm., 6, 547 (1978). 